\title{
Equivalent Representations of Non-Exponential Discounting Models*
}

\author{
James Feigenbaum ${ }^{\dagger}$ \\ Utah State University
}

December 20, 2015

\begin{abstract}
I characterize the entire class of consumption rules for finite-horizon models in which consumption is proportional to lifetime wealth. Any such rule can be obtained from a preference model with CRRA period utility. In a steady state with constant interest rates, a proportional consumption rule can be derived from a model with time-consistent preferences or from a model with possibly time-inconsistent preferences in which a household continually reoptimizes future utility discounted relative to the present instant. These two preference models will only coincide for the special case when the discount function is exponential. More generally, there will be two distinct yet observationally equivalent preference models. I argue that the time-consistent model can be used to do welfare analysis for the equivalent non-exponential discounting model. Under this welfare metric, Social Security cannot improve welfare for any non-exponential discounting model if the internal rate of return is negative. Applying the equivalence in the opposite direction, hyperbolic-like discounting may arise because that is a simpler way for the brain to process a standard exponential discount function after accounting for mortality risk.

JEL Classification: D11, D63, E21

Keywords: consumption, saving, naive hyperbolic discounting, exponential discounting, Social Security, welfare analysis, myopia, mortality risk, behavioral economics, lifecycle model
\end{abstract}

Though it is not as mysterious as quantum mechanics would have appeared to a 19th-Century physicist, to someone trained in neoclassical economics the hyperbolic discounting model is still a strange beast. One offputting aspect is the multiplicity of selves that inhabit the model, which makes welfare analysis seem completely arbitrary. In sophisticated versions of the model where different selves play games with each other, the model becomes even more absurd-not to mention overly complicated. While I can testify to reoptimizing after discovering I have made a mistake, I cannot say I have ever successfully committed to a future behavior that appears optimal in the present but suboptimal in the future. In my experience the future self always gets its way when the future arrives.

Despite the aesthetic distaste that many economists have for hyperbolic discounting, the accumulated evidence from experiments suggests that Samuelson's (1937) exponential discounting model, which he explicitly designed for mathematical simplicity and not because he had any data whatsoever to support it, does a poor job of fitting people's consumption choices (Frederick, Loewenstein, and O'Donoghue (2002) and Rabin (2002)). Laibson (1997) followed up this psychologial research, convincing many economists that hyperbolic

*I would like to thank Hengjie Ai, Frank Caliendo, Rick Evans, Scott Findley, Aspen Gorry, Nick Guo, Thomas Jeitschko, Miles Kimball, Val Lambson, Amy Odum, Kirk Phillips, and participants at the BYU/USU macro and Midwest Theory workshops for their suggestions and comments.

${ }^{\dagger}$ Corresponding author: J.Feigen@aggiemail.usu.edu. 
discounting can account for several puzzles in the consumption and saving literature. The hyperbolic model also has its critics though, for psychologists have found that, while it works quite well for modeling animal behavior, additional parameters are needed to fit human behavior (Myerson and Green (1995)). However, such data merely points to more refined non-exponential discounting models and not a return to Samuelson's model.

Strotz (1956) was the first to explore relative discounting models in which households, rather than valuing consumptions with a constant metric that depends only on the absolute times of these consumptions, value future consumptions based on the time delay before each consumption occurs. Whereas the valuation of consumption streams with an absolute discounting function is invariable so the optimal stream can be chosen once and forever more at age 0 , with a relative discounting function the valuation at different times can be different. Indeed, Strotz showed that, unless the discounting function is exponential, plans for future behavior will be time-inconsistent, and households will have to reoptimize at every instant. ${ }^{1}$ This implies that non-exponential relative discounting functions can yield preference reversals: when valuing consumption streams that begin at some distant time in the future, a household may rank the consumption streams differently now than it would later at the start of these consumption streams.

Since the rational paradigm depends crucially on the assumption that individuals know how to consistently rank consumption allocations, evidence for a non-exponential relative discounting function is often viewed as evidence for bounded rationality. Here I show that, in a steady state where interest rates are constant, any time-inconsistent preference model with constant relative risk aversion (CRRA) period utility can be mapped into an observationally equivalent time-consistent preference model that yields the same consumption rule, and vice versa. Thus, behavior that is consistent with non-exponential discounting will also be consistent with time-consistent preferences that are in no way irrational.

For example, the behavior of a myopic household that discounts exponentially but only values its consumption over a short horizon can be replicated by a time-consistent preference model in which the discount function is piecewise exponential with two pieces. During the early piece the value of consumption will fall off quite rapidly, mimicking the myopic household's belief that it should compact a whole lifetime's worth of consumption into the shorter period that it cares about and thus rapidly dissipate its wealth. The later piece applies when the myopic household sees the true end of its life, after which the discount functions for the two equivalent households decay at the same rate.

Corresponding time-consistent and time-inconsistent discounting models are not observationally equivalent under any and all circumstances. In the present environment, the household must choose how to allocate consumption given an exogenous income stream, which is the sort of economic problem a household faces in reality (ignoring labor and investment decisions). When confronted with hypothetical experiments where one can choose between different consumption streams that only diverge in the future, the time consistency or inconsistency of the discounting model can be identified. The relative discounting model can exhibit preference reversals, whereas the time-consistent discounting model cannot. ${ }^{2} \quad$ It is important, however, to recognize that experiments that can elicit preference reversals are largely artificial. Choices between future consumption streams that only diverge in the future rarely arise in nature. It is very common to face a situation analogous to having to choose between getting one cookie right now or two cookies tomorrow, but it is very unusual for a person to have to commit to a choice between getting one cookie tomorrow or two cookies the day after tomorrow. Experiments involving the second type of choice are useful for discovering how the brain processes comparisons of consumption streams, but that may be all they are useful for in terms of positive economics.

\footnotetext{
${ }^{1}$ We only consider the case where households are naive about the time-inconsistency of their preferences, so they do not anticipate that they will reoptimize in the future or, indeed, the next instant. If utility is logarithmic, sophisticated and naive households will behave the same, so our results do extend to the sophisticated case then (Marín-Solano and Navas, (2009)).

${ }^{2}$ One can observe preference reversals even with time-consistent preferences if the experimenter is not careful to fix the consumption stream that occurs during the intervening period between the times when the respondent is asked to rank ensuing consumption streams (Noor (2009)).
} 
My equivalence result has two implications. First, since the behavior that comes from a non-exponential relative discounting function can always be replicated by a time-consistent, though still non-exponential, discounting function, non-exponential discounting is at worst a benign form of bounded rationality. The extensive debate about whether households are better modeled as having time-consistent or time-inconsistent preferences is academic. We can use whichever representation is more appropriate under the circumstances. This leads to the second result: the equivalent time-consistent model provides a way of measuring welfare for a model with a non-exponential relative discounting function that is not based on an arbitrary weighting of the valuations of different selves. ${ }^{3}$

I then use the equivalent time-consistent model to study the partial-equilibrium, welfare effects of Social Security in a model with non-exponential discounting. This yields in a simpler fashion the result of İmrohoroğlu, İmrohoroğlu, and Joines (2003) and Caliendo (2011) that nonexponential discounting cannot justify Social Security on the grounds that it improves welfare. For any proportional consumption rule, lifetime welfare is a strictly increasing function of the present value of expected income over the lifecycle. If the internal rate of return of Social Security is negative (as it presently is for the United States), it cannot increase the relevant time-consistent measure of utility.

Barro (1999) established an observational equivalence between the exponential model and the sophisticated hyperbolic model for infinite-horizon growth models. Caliendo and Findley (2013) have done the same for the naive model with log utility in a lifecycle context. I generalize the latter result. In a finitehorizon model, any preference specification with a period utility function that exhibits constant relative risk aversion will yield a consumption rule in which consumption is proportional to the present value of lifetime wealth. This is true both for time-consistent models and for possibly time-inconsistent relative discounting models. My principal finding is that, in a steady state with constant interest rates, the mapping from either type of preference model to the corresponding consumption rule is invertible. Consequently, there is a one-to-one mapping between time-consistent preference models and relative discounting models. In a dynamic setting with perfect foresight about interest rates, the mapping from a relative discounting model to a time-consistent preference model will still exist, though the inverse mapping may not exist. ${ }^{4}$

This one-to-one mapping only depends on preference parameters and the interest rate. Thus the equivalent time-consistent model can, in general, be used to evaluate the welfare effects of policy changes that only alter a household's income stream without impacting the interest rate. For example, we can use this approach to study changes in tax policy or Social Security in partial equilibrium. For the special case of log utility, the caveat that we can only use the mapping for welfare analysis in partial equilibrium also goes away. Another policy experiment that we can evaluate is the purported benevolence of government programs that help households with time-inconsistent preferences commit to plans formed early in life. I find that such interventions can only reduce the equivalent time-consistent measure of lifetime utility.

In a calibrated model where I match the observed capital-labor ratio of the US economy, I compute the time-consistent discounting function that replicates naive hyperbolic and myopic discounting models. Contrary to popular wisdom, the hyperbolic model actually leads to more initial saving and less initial consumption than an exponential model that produces the same capital-labor ratio. Relative to the exponential model, the time-consistent form of the hyperbolic model puts more weight on consumption in the middle of life. The myopic model, on the other hand, behaves as one would expect, placing very high weight on consumption early in life relative to consumption later in life.

Conversely, in a standard exponential-discounting model augmented with uninsurable mortality risk, the effective discount function, though time-consistent, will generally not be exponential. The exponential discount function coming from preferences will be mixed in with the survival probability, which will only

\footnotetext{
${ }^{3}$ For some readers, the argument that this is the only known way to evaluate welfare in a non-arbitrary fashion may be sufficient to justify using the equivalent time-consistent preference model to evaluate welfare. For those who are not persuaded by this purely practical consideration, we provide a more compelling, biological argument at the end of the paper.

${ }^{4}$ It will exist if the period utility function is logarithmic.
} 
be exponential in the counterfactual case of a constant hazard rate of dying In computing the equivalent relative discounting function, I find that a hyperbolic discounting model is a much closer fit to this relative discounting function than an exponential discounting model. Other biological considerations not accounted for in the standard model, such as the drive to procreate, may further distort both the time-consistent and corresponding relative discounting functions away from a pure exponential.

While time-consistent preferences have many advantages that make them a convenient modeling choice for economists, they are not necessarily so amenible for the programming of biological organisms. To employ a time-consistent discounting function, one has to determine the age when consumption will occur, and the discounting function may vary considerably depending on that age. With a relative discounting function, in contrast, only the length of the time delay preceding the consumption matters. Some common consumption comparisons will occur repeatedly throughout the lifecycle, and the same relative discounting function can be used for these comparisons regardless of whether the consumer is young, middle-aged, or old. Human beings may exhibit time-inconsistent preferences because it is less complicated for the brain to process decisions with an age-independent relative discounting function.

The paper is organized as follows. Section 1 describes the environment in which we will explore these different consumption rules. Section 2 introduces the concept of a proportional consumption rule. In Section 3, I show how exponential, hyperbolic, and myopic discounting fit into the proportional consumption rule framework. In Section 4, I establish that any proportional consumption rule can be derived from a time-consistent discounting function. Likewise in Section 5, I establish that if interest rates are constant than any proportional consumption rule can be derived from a relative discounting function. In Section 6, I present numerical examples of equivalent discounting functions. In Section 7, I show that Social Security cannot improve welfare if it decreases the present value of income. I conclude in Section 8 and discuss how I interpret these results.

\section{The Environment}

First, let us establish the budgetary constraints facing a household, which we will hold fixed across all the preference models that we consider below. A household lives with certainty to age $T$. At age $t$, it receives the flow of labor or transfer income $y(t) \geq 0$, which must be positive on a subset of positive measure of $[0, T]$. This income can be consumed $c(t)$ or saved as $k(t)$ at the instantaneous interest rate $r(t)$. Thus the household must choose a path of $k(t)$ and $c(t)$ subject to the budget constraint

$$
\frac{d k(t)}{d t}=y(t)+r(t) k(t)-c(t)
$$

and the boundary conditions

$$
k(0)=k(T)=0 .
$$

Let us define

$$
R(t)=\exp \left(\int_{0}^{t} r(s) d s\right)
$$

which is the gross return due to interest compounding between ages 0 and $t .^{5}$ Note that

$$
\frac{d R(t)}{d t}=r(t) \exp \left(\int_{0}^{t} r(s) d s\right)=r(t) R(t)
$$

We can rewrite (1) as

$$
\frac{d}{d t}\left(\frac{k(t)}{R(t)}\right)=\frac{y(t)-c(t)}{R(t)}
$$

\footnotetext{
${ }^{5}$ Throughout the paper, we will assume all relevant functions are suitably well-behaved so they can be integrated.
} 
Integrating this from $t$ to $T$, and using the terminal condition $k(T)=0$, we obtain

$$
k(t)=\int_{t}^{T} \frac{R(t)}{R(s)}[c(s)-y(s)] d s .
$$

Given the path of current and future consumption $c(s)$ for $s \in[t, T]$, we can determine the saving $k(t)$ at age $t$.

If we define lifetime wealth as

$$
W(t)=\int_{t}^{T} \frac{R(t)}{R(s)} y(s) d s+k(t),
$$

we can rewrite $(6)$ as

$$
\int_{t}^{T} \frac{R(t)}{R(s)} c(s) d s=W(t) .
$$

\section{General Proportional Consumption Rules}

How is consumption determined? As in most of the economic literature, we will assume here that consumption is chosen based on preferences. However, we are going to reverse the common methodology of deriving consumption from an assumed preference model. Instead, we will assume consumption is observed and then try to back out what preferences are consistent with these observations. Following Feigenbaum, Caliendo, and Gahramanov (2011), we consider consumption rules that are feasible in the environment of Section 1.

Specifically, I focus on rules such that consumption at a given age $t$ is proportional to total lifetime wealth at that age:

$$
c(t)=m(t) W(t),
$$

where the proportionality factor $m(t)>0$ is the marginal propensity to consume (MPC) out of lifetime wealth at age $t .{ }^{6}$ What conditions do we need to impose on the function $m(t)$ to ensure the budget constraint (1) and boundary conditions (2) will be satisfied? Surprisingly little. If we substitute (9) into the budget constraint to obtain a differential equation for $k(t)$

$$
\frac{d k(t)}{d t}=y(t)+r(t) k(t)-m(t)\left[\int_{t}^{T} \frac{R(t)}{R(s)} y(s) d s+k(t)\right],
$$

we can solve for $k(t)$ given the initial condition $k(0)=0$. We then only need to verify that the terminal condition $k(T)=0$ holds.

Proposition 1 A solution to (10) with $k(0)=0$ will satisfy $k(T)=0$ as long as

$$
\lim _{t \rightarrow T} m(t)(T-t)=1 .
$$

Eq. (11) is the continuous-time analog of the usual result in discrete-time models that a household must consume all its remaining wealth in the last period. In continuous time, as the household approaches its end, it will plan to consume its remaining wealth so $W(T)=0$. If the path of $W(t)$ is differentiable, then as $t \rightarrow T$, we must have $W(t) \approx C(T-t)$ for some constant $C>0$. The condition (11) then implies that $c(t) \approx C$ as $t \rightarrow T$. The proof follows.

\footnotetext{
${ }^{6}$ Note that consumption is proportional in the sense that, at a given age $\mathrm{t}$, the fraction of lifetime wealth consumed at $\mathrm{t}$ will be the same regardless of whether the household is rich or poor. However, the fraction consumed is not assumed to be constant over the lifecycle.
} 
Analogous to $R(t)$ and $r(t)$, let us define

$$
M(t)=\exp \left(\int_{0}^{t} m(s) d s\right)
$$

which satisfies

$$
\frac{d M(t)}{d t}=m(t) M(t)
$$

Since

$$
\frac{d}{d t}\left(\frac{M(t)}{R(t)} k(t)\right)=\frac{M(t)}{R(t)}\left[\frac{d k(t)}{d t}+(m(t)-r(t)) k(t)\right]
$$

we can rewrite (10) as

$$
\frac{d}{d t}\left(\frac{M(t)}{R(t)} k(t)\right)=\frac{M(t)}{R(t)}\left[y(t)-m(t) \int_{t}^{T} \frac{R(t)}{R(s)} y(s) d s\right] .
$$

Integrating this from $t=0$ to $T$, we obtain the condition

$$
k(T)=\frac{R(T)}{M(T)} \int_{0}^{T} \frac{M(t)}{R(t)}\left[y(t)-m(t) \int_{t}^{T} \frac{R(t)}{R(s)} y(s) d s\right] d t=0,
$$

which must be satisfied for any choice of $y(t)$ and $r(t)$ if $m(t)$ is a valid MPC. It will be satisfied if

$$
\frac{M(t)}{M(T)}=0
$$

for all $t<T$. That is if

$$
\int_{t}^{T} m(s) d s=\infty
$$

for all $t<T$. Eq. (11) is a sufficient condition for this to hold. ${ }^{7}$ Thus any positive MPC function $m(t)$ that satisfies (11) will define a proportional consumption rule.

Note that consumption will always be positive under such a rule. Inserting (7) into (9) yields

$$
c(t)=m(t)\left[\int_{t}^{T} \frac{R(t)}{R(s)} y(s) d s+k(t)\right] .
$$

Differentiating the log of (17) gives

$$
\begin{aligned}
\frac{d \ln c(t)}{d t} & =\frac{d \ln m(t)}{d t}+\frac{d}{d t} \ln \left[\int_{t}^{T} \frac{R(t)}{R(s)} y(s) d s+k(t)\right] \\
& =\frac{d \ln m(t)}{d t}+\frac{r(t) \int_{t}^{T} \frac{R(t)}{R(s)} y(s) d s-y(t)+\frac{d k(t)}{d t}}{\int_{t}^{T} \frac{R(t)}{R(s)} y(s) d s+k(t)} .
\end{aligned}
$$

Using (1) and (9), this simplifies to

$$
\frac{d \ln c(t)}{d t}=\frac{d \ln m(t)}{d t}+\frac{r(t) \int_{t}^{T} \frac{R(t)}{R(s)} y(s) d s+r(t) k(t)-m(t) W(t)}{\int_{t}^{T} \frac{R(t)}{R(s)} y(s) d s+k(t)}
$$

\footnotetext{
${ }^{7}$ Eq. (11) is actually a stronger condition than what is necessary to guarantee (15). However, (11) is satisfied by all the consumption rules that we consider and has the reasonable economic interpretation discussed above.
} 
which reduces to

$$
\frac{d \ln c(t)}{d t}=\frac{d \ln m(t)}{d t}+r(t)-m(t) .
$$

Integrating this from 0 to $t$, we get

$$
\ln c(t)-\ln c(0)=\ln m(t)-\ln m(0)+\int_{0}^{t}(r(s)-m(s)) d s
$$

Since $k(0)=0$, we have

$$
c(0)=m(0) \int_{0}^{T} \frac{y(t)}{R(t)} d t .
$$

Exponentiating (19), and using (3), (12), and (20) obtains

$$
c(t)=m(t) \frac{R(t)}{M(t)} \int_{0}^{T} \frac{y(s)}{R(s)} d s,
$$

which is positive given our assumptions about $m(t), r(t)$, and $y(t)$. Thus if a household follows a proportional consumption rule, its consumption at any age will be proportional to the age- 0 value of its lifetime income stream.

\section{Standard Discounting Models}

Now let us see how three standard discounting models fit into the framework of Section 2: exponential discounting, naive hyperbolic discounting, and myopic preferences.

\subsection{Exponential Discounting}

The most frequently used discounting model is Samuelson's (1937) exponential discounting model in which the household chooses $c(t)$ to maximize

$$
\int_{0}^{T} \exp (-\rho t) u(c(t)) d t
$$

subject to (1) and (2). See Appendix A.1 for details on the solution to this model. As is well known, the optimal consumption profile with these preferences allocates consumption over the lifecycle so the growth rate of marginal utility is $\rho-r(t)$. This result is the continuous-time analog of setting the marginal rate of substitution between consumption at $t$ and $t+d t$ equal to the relative price of $t+d t$ consumption in terms of time- $t$ consumption. Given $c(t)$, this implies the consumption profile

$$
c(s)=\left(u^{\prime}\right)^{-1}\left(u^{\prime}(c(t)) \exp (\rho(s-t)) \frac{R(t)}{R(s)}\right)
$$

for $s \in[t, T]$. Inserting the consumption profile into (8) yields an equation that implicitly determines $c(t)$ as a function of lifetime wealth $W(t)$.

$$
\int_{t}^{T} \frac{R(t)}{R(s)}\left(u^{\prime}\right)^{-1}\left(u^{\prime}(c(t)) \exp (\rho(s-t)) \frac{R(t)}{R(s)}\right) d s=W(t) .
$$


For most choices of the period utility function $u(c),(24)$ cannot be solved to obtain an analytic expression for $c(t)$. From now on we will focus on the special case of CRRA utility ${ }^{8}$

$$
u(c ; \gamma)=\left\{\begin{array}{cc}
\ln c & \gamma=1 \\
\frac{1}{1-\gamma} c^{1-\gamma} & \gamma \neq 1
\end{array}\right.
$$

which satisfies

$$
u^{\prime}(c ; \gamma)=c^{-\gamma} .
$$

Eq. (23) then simplifies to

$$
c(s)=\left(\frac{R(t)}{R(s)} \exp (\rho(s-t))\right)^{-\frac{1}{\gamma}} c(t)
$$

and Eq. (24) to

$$
c_{\exp }(t ; \rho)=\frac{W(t)}{\int_{t}^{T}\left(\frac{R(t)}{R(s)}\right)^{\frac{\gamma-1}{\gamma}} \exp \left(-\frac{\rho}{\gamma}(s-t)\right) d s} .
$$

Thus the exponential discounting model with CRRA utility gives a proportional consumption rule with MPC

$$
m_{\exp }(t ; \rho)=\frac{1}{\int_{t}^{T}\left(\frac{R(t)}{R(s)}\right)^{\frac{\gamma-1}{\gamma}} \exp \left(-\frac{\rho}{\gamma}(s-t)\right) d s} .
$$

In the special case of $\gamma=1, R(t)$ cancels from the integral in the denominator, and the MPC reduces to

$$
m_{\exp }(t ; \rho)=\frac{\rho}{1-\exp (-\rho(T-t))},
$$

which is purely a function of the preferential discount rate $\rho$. The MPC then also has the interpretation of being the reciprocal of the area of the discount function over the remaining lifespan, $\int_{t}^{T} \exp (-\rho s) d s .^{9}$

\subsection{Naive Hyperbolic Discounting}

Next we consider a naive hyperbolic discounter. At age $t$, he discounts consumption at age $s>t$ by $\frac{1}{1+\eta(s-t)}$ for $\eta>0$. Given $k(t)$, the hyperbolic discounter at age $t$ will plan his future consumption $c(s ; t)$ and asset holdings $k(s ; t)$ at age $s \in[t, T]$ so as to maximize

$$
U(t)=\max \int_{t}^{T} \frac{u(c(s ; t) ; \gamma)}{1+\eta(s-t)} d s
$$

subject to the imagined budget constraint

$$
c(s ; t)+\frac{\partial k(s ; t)}{\partial s}=y(s)+r(s) k(s ; t)
$$

and the boundary conditions

$$
k(t ; t)=k(t)
$$

\footnotetext{
${ }^{8}$ Any period utility function in the hyperbolic absolute risk aversion (HARA) class will allow an analytic solution to (24), but for other utility functions in this class the consumption rule will be affine rather than strictly proportional.

${ }^{9}$ For the special case of $\log$ utility, this is a general property of the MPC across discounting models (Findley and Caliendo (2013)).
} 


$$
k(T ; t)=0 .
$$

These constraints assume the household sticks to its age- $t$ plan. However, as Strotz (1956) showed, only for an exponential discounting function $\exp (-\rho(s-t))$ will the household make the same plans for its future consumption $c(s ; t)=c\left(s ; t^{\prime}\right)$ at different ages $t, t^{\prime}<s$. Actual consumption at age $t$ must always follow the plan set at $t: c(t)=c(t ; t)$. However, since the household is naive about its hyperbolic discounting, in the next instant $t^{\prime}$ it will revise its plans, so $c\left(t^{\prime}\right)=c\left(t^{\prime} ; t^{\prime}\right) \neq c\left(t^{\prime} ; t\right)$. Asset holdings $k(t)$ must satisfy the actual budget constraint (1), consistent with the path of actual consumption $c(t)$.

The optimal consumption profile with naive hyperbolic discounting is analogous to (27) for the exponential model, replacing the latter's discount function $\exp (-\rho t)$ with the hyperbolic discount function $(1+\eta(s-t))^{-1}$ :

$$
c(s ; t)=\left(\frac{R(s)}{R(t)(1+\eta(s-t))}\right)^{1 / \gamma} c(t) .
$$

Details of the solution are in Appendix A.2.

Analogous to the actual budget constraint, the imagined budget constraint (32) can be rewritten

$$
\frac{\partial}{\partial s}\left(\frac{k(s ; t)}{R(s)}\right)=\frac{y(s)-c(s ; t)}{R(s)},
$$

which yields the imagined lifetime budget constraint

$$
\int_{t}^{T} \frac{R(t)}{R(s)} c(s ; t) d s=W(t) .
$$

Inserting (35) into (36), actual consumption $c(t)$ at age $t$ is

$$
c_{\text {hyp }}(t ; \eta)=\frac{W(t)}{\int_{t}^{T}\left(\frac{R(s)}{R(t)}\right)^{\frac{1-\gamma}{\gamma}}[1+\eta(s-t)]^{-1 / \gamma} d s} .
$$

This is a proportional consumption rule with MPC

$$
m_{\text {hyp }}(t ; \eta)=\frac{1}{\int_{t}^{T}\left(\frac{R(s)}{R(t)}\right)^{\frac{1-\gamma}{\gamma}}[1+\eta(s-t)]^{-1 / \gamma} d s} .
$$

In the special case when $\gamma=1,(38)$ reduces to

$$
m_{\text {hyp }}(t ; \eta)=\frac{\eta}{\ln (1+\eta(T-t))} .
$$

As in the exponential case, this MPC is the reciprocal of the integral of the, now relative, discount function over the remaining lifespan: $\int_{t}^{T} \frac{d s}{1+\eta(s-t)}$.

For comparison with the exponential MPC, note that since $\exp (\eta x)$ is strictly concave

$$
\exp (\eta x) \geq 1+\eta x
$$

with equality only if $x=0$. Since $\exp \left(-\frac{\eta}{\gamma}(s-t)\right) \leq(1+\eta(s-t))^{-1 / \gamma}$, comparing (29) to (38), we see that $m_{\text {hyp }}(t ; \eta)<m_{\exp }(t ; \eta)$ for $t<T$.

Others (Salanie and Treich (2006)) have pointed this out before, but it is worth mentioning that for a given value of wealth the hyperbolic discounter actually saves more than the exponential discounter when 
$\eta=\rho$. In other words, if we define patience to be the willingness to defer consumption to later times, a hyperbolic discounter will be more patient than an exponential discounter with the same "discount rate". ${ }^{10}$ While $\eta$ and $\rho$ are both measures of patience, they have different definitions and different quantitative properties. ${ }^{11}$ The exponential model is characterized by having a constant $\rho$ whereas the hyperbolic model has a constant $\eta$. If we compute the decay rate of $(1+\eta t)^{-1}$, the variable analogous to $\rho$ for the hyperbolic model, this is

$$
\frac{\eta}{1+\eta t} \leq \eta
$$

so if $\eta=\rho$ the hyperbolic discounter will be more patient than the exponential discounter. ${ }^{12}$ When I calibrate the models below, I will use an invariant measure of patience, aggregate saving over the lifecycle, which also has the advantage of being observable.

\subsection{Myopic Preferences}

Under myopic preferences, the household will only value utility within a limited time horizon $H>0$. Assuming discounting is still exponential within this time horizon, a household at age $t$ will maximize

$$
\int_{t}^{\min \{t+H, T\}} \exp (-\rho(s-t)) u(c(s ; t)) d s
$$

subject to the imagined constraints (32), (33), and (34). Note that this differs from the short-term horizon models of Caliendo and Aadland (2007), Findley and Caliendo (2013), and Park (2009) because the household here perceives that it will earn income after $t+H$. It just does not value its consumption after $t+H$. Consequently, the imagined consumption profile for $s \in[t, T]$ is the same as (27) except that the household plans to stop consuming at the edge of its current time horizon:

$$
c(s ; t)=\left\{\begin{array}{cc}
c(t)\left(\frac{R(s)}{R(t)}\right)^{1 / \gamma} \exp \left(-\frac{\rho}{\gamma}(s-t)\right) & s \in[t, \min \{t+H, T\}] \\
0 & s \in(t+H, T]
\end{array} .\right.
$$

Details of the solution are given in Appendix A.3.

As in 3.2, actual age- $t$ consumption $c(t)$ is determined by the imagined lifecycle budget constraint (36)

$$
c_{m y o}(t ; \rho, H)=\frac{W(t)}{\int_{t}^{\min \{t+H, T\}}\left(\frac{R(s)}{R(t)}\right)^{\frac{1-\gamma}{\gamma}} \exp \left(-\frac{\rho}{\gamma}(s-t)\right) d s} .
$$

The myopic MPC is

$$
m_{m y o}(t ; \rho, H)=\frac{1}{\int_{t}^{\min \{t+H, T\}}\left(\frac{R(s)}{R(t)}\right)^{\frac{1-\gamma}{\gamma}} \exp \left(-\frac{\rho}{\gamma}(s-t)\right) d s} .
$$

\footnotetext{
${ }^{10}$ Some may find this counterintuitive since hyperbolic discounting is often cited as an explanation for undersaving. The notion that hyperbolic discounters are intrinsically less patient than exponential discounters comes from naively interpreting the results from a popular calibration of quasihyperbolic discounting models. The quasihyperbolic discounting model approximates hyperbolic discounting in discrete time by introducing an extra discount factor between the current period and all following periods. Quasihyperbolic and exponential discount factors coincide if this extra discounting is eliminated. Consequently, many papers compare a quasihyperbolic model with this extra discounting to the corresponding exponential model without it. Since the overall discount function in the quasihyperbolic model is then always smaller than in the exponential model, this assumes by construction that a quasihyperbolic discounter is less patient than an exponential discounter.

${ }^{11}$ In the notation introduced in Section $5, \rho=-\ln D_{r}(t) / d t$, i.e. $\rho$ is the decay rate of the relative discount function, whereas $\eta=(d / d t)\left(D_{r}(t)^{-1}\right)$, i.e. $\eta$ is the derivative of the inverse relative discount function.

${ }^{12}$ Conversely, the derivative of the inverse of the relative discounting function for the exponential model is $\rho \exp (\rho t) \geq \rho$, so the hyperbolic discounter is also more patient than the exponential discounter with this alternative measure of patience.
} 
For the case when $\gamma=1,(43)$ reduces to

$$
m_{\text {myo }}(t ; \rho, H)=\frac{\rho}{1-\exp (-\rho \min \{H, T-t\})}
$$

When $t>T-H, m_{m y o}(t ; \rho, H)=m_{\exp }(t ; \rho, H)$, so the myopic and exponential MPCs are the same at late ages if the preferential discount rate is the same. For $t<T-H$,

$$
\begin{aligned}
m_{\text {myo }}(t ; \rho, H) & =\frac{1}{\int_{t}^{\min \{t+H, T\}}\left(\frac{R(s)}{R(t)}\right)^{\frac{1-\gamma}{\gamma}} \exp \left(-\frac{\rho}{\gamma}(s-t)\right) d s} \\
& >\frac{1}{\int_{t}^{T}\left(\frac{R(s)}{R(t)}\right)^{\frac{1-\gamma}{\gamma}} \exp \left(-\frac{\rho}{\gamma}(s-t)\right) d s}=m_{\exp }(\rho),
\end{aligned}
$$

so the myopic MPC is larger early in life. This is because the myopic household believes it has the same income stream to distribute over a shorter lifespan. If the discount rate parameter is the same, the myopic household consumes more than the exponential discounter who consumes more than the hyperbolic consumer. $^{13}$

\section{General Time-Consistent Discounting Model}

We now generalize the exponential discounting model of Section 3.1 and suppose that households choose the path $c(t)$ to maximize

$$
U=\int_{0}^{T} D(t) u(c(t) ; \gamma) d t
$$

subject to (1) and (2) for some discount function $D(t):[0, T] \rightarrow \mathbf{R}_{++}$. Noor (2009) argues that a general time-consistent discounting model can produce behavior consistent with hyperbolic discounting. Here I show that any proportional consumption rule will have a discount function for which that consumption rule is the optimal behavior. Indeed, there is a 1-1 correspondence between discount functions and proportional consumption rules.

With utility (45), the optimal consumption profile for $s \in[t, T]$ is

$$
c(s)=c(t)\left(\frac{D(s)}{D(t)} \frac{R(s)}{R(t)}\right)^{1 / \gamma},
$$

which generalizes $(23)$ for the case when $D(t)$ is not restricted to the exponential form $\exp (-\rho t) .{ }^{14}$ Inserting this in (8),

$$
c(t)=\frac{W(t)}{\int_{t}^{T}\left(\frac{D(s)}{D(t)}\left(\frac{R(s)}{R(t)}\right)^{1-\gamma}\right)^{1 / \gamma} d s} .
$$

Thus the MPC for the general discounting function is

$$
m(t)=\frac{1}{\int_{t}^{T}\left(\frac{D(s)}{D(t)}\left(\frac{R(s)}{R(t)}\right)^{1-\gamma}\right)^{1 / \gamma} d s} .
$$

\footnotetext{
${ }^{13}$ We will discuss how we would propose calibrating these three models in Section 6 .

${ }^{14}$ For details, see Appendix B
} 
My first major result is to show that, for a given MPC function $m(t)$, we can back out of Eq. (48) the discount function $D(t)$ that yields that MPC as part of the optimal consumption rule. ${ }^{15}$

Proposition 2 Let $m:[0, T] \rightarrow \mathbf{R}_{++}$satisfy (11) so it is the MPC for a proportional consumption rule. There exists $D:[0, T] \rightarrow \mathbf{R}_{++}$such that $D(0)=1$ and Eq. (48) holds.

Let $m(t)$ be the MPC for a proportional consumption rule. Differentiating (48) gives

$$
\begin{aligned}
\frac{d m(t)}{d t} & =-\frac{-\left(\frac{D(t)}{D(t)}\left(\frac{R(t)}{R(t)}\right)^{1-\gamma}\right)^{1 / \gamma}+\int_{t}^{T} \frac{\partial}{\partial t}\left(\frac{D(s)}{D(t)}\left(\frac{R(s)}{R(t)}\right)^{1-\gamma}\right)^{1 / \gamma} d s}{\left[\int_{t}^{T}\left(\frac{D(s)}{D(t)}\left(\frac{R(t)}{R(t)}\right)^{1-\gamma}\right)^{1 / \gamma} d s\right]^{2}} \\
& =m(t)^{2}\left[1+\frac{1}{\gamma}\left(\frac{d \ln D(t)}{d t}+(1-\gamma) r(t)\right) \int_{t}^{T}\left(\frac{D(s)}{D(t)}\left(\frac{R(t)}{R(t)}\right)^{1-\gamma}\right)^{1 / \gamma} d s\right]
\end{aligned}
$$

Using (48) again, this simplifies to

$$
\frac{d \ln m(t)}{d t}=m(t)+\frac{1}{\gamma}\left[\frac{d \ln D(t)}{d t}+(1-\gamma) r(t)\right] .
$$

Solving (49) for the growth rate of $D(t)$, we obtain a first-order differential equation for $D(t)$ :

$$
\frac{d \ln D(t)}{d t}=\gamma\left[\frac{d \ln m(t)}{d t}-m(t)\right]+(\gamma-1) r(t)
$$

Normalizing $D(0)=1$,

$$
\ln D(t)=\int_{0}^{t}\left(\gamma\left[\frac{d \ln m(s)}{d t}-m(s)\right]+(\gamma-1) r(s)\right) d s .
$$

Using (3) and (12), this simplifies to

$$
D(t)=\left(\frac{m(t)}{m(0)}\right)^{\gamma} \frac{R(t)^{\gamma-1}}{M(t)^{\gamma}}
$$

Note that the correspondence between $m(t)$ and the discount rate in (51) holds regardless of the income path. This fact will be essential to the later interpretation of this result. In contrast, the determination of the discount function does depend on the path of interest rates. A household maximizing a utility function of the form (45) will only care about its income path to the extent that its income path determines its budget set, and lifetime wealth is a sufficient statistic containing all the information in the income path that is relevant to determining the size of the budget set. The relationship between $D(t)$ and $m(t)$ is ultimately a description of how the optimal consumption rule depends on the budget set. Since the interest rate determines the gradient of the budget set frontier, the relationship between $D(t)$ and $m(t)$ also depends on the path of $r(t)$ except when utility is logarithmic. In that special case of $\gamma=1$, income and substitution

\footnotetext{
${ }^{15}$ Similar results would obtain for other HARA utility functions, though in general the consumption function will be affine in wealth. We focus on CRRA utility to simplify the discussion since in that case we only have one coefficient, the MPC, to relate to preferences.
} 
effects stemming from variation of the interest rate exactly cancel, so the optimal consumption rule only depends on the size of the budget set and not the slope of its frontier.

For the case of myopic discounting with $\gamma=1$, there is an analytic expression for the equivalent timeconsistent discount function

$$
D_{m y o}(t ; \rho, H)=\left\{\begin{array}{cc}
\exp \left(-\frac{\rho t}{1-\exp (-\rho H)}\right) & t<T-H \\
\exp \left(-\rho t-\frac{\rho(T-H) \exp (-\rho H)}{1-\exp (-\rho H)}\right) & t>T-H
\end{array} .\right.
$$

For $t>T-H$, the household's time horizon is longer than its remaining life so it behaves as though it is an exponential discounter with discount rate $\rho$. At earlier times, it still behaves as though it is an exponential discounter but with the effective discount rate

$$
\rho_{\text {eff }}=\frac{\rho}{1-\exp (-\rho H)}>\rho
$$

For the case of hyperbolic discounting, there is no analytic expression for the equivalent time-consistent discount function. In Section 6, I will compute the effective discount function numerically.

\section{$5 \quad$ Relative Discounting Model}

We can also generalize the hyperbolic model of 3.2 and the myopic model of 3.3 to consider time-inconsistent models with any relative discounting function. Let $D_{r}:[0, T] \rightarrow \mathbf{R}_{++}$such that $D_{r}(0)=1$ be a relative discount function so that $D_{r}(s)$ measures how utility is discounted after a delay $s$. A household at age $t$ with assets $k(t)$ will maximize

$$
U_{r}(t, k(t))=\max \int_{t}^{T} D_{r}(s-t) u(c(s) ; \gamma) d s
$$

subject to the imagined constraints (32), (33), and (34).

There is no difference in the assumptions we make about the discount functions $D(t)$ in Section 4 and the assumptions we make about relative discount functions here. The same function could be used in both classes of model. What is different is the optimization problems (45) and (54). Only for an exponential function will the two problems have the same solution. Thus the exponential discounting model can be viewed equivalently as either a time-consistent discounting model or a relative discounting model.

As in Section 4, where we replaced $\exp (-\rho(s-t))$ by $D(s) / D(t)$, here we generalize the consumption function of the exponential model by replacing $\exp (-\rho(s-t))$ in $(23)$ with $D_{r}(s-t) .{ }^{16}$ That is, at age $t$ the household will plan to follow the consumption path

$$
c(s)=c(t)\left(\frac{R(s)}{R(t)} D_{r}(s-t)\right)^{1 / \gamma}
$$

Inserting this into the lifecycle budget constraint from $t$ to $T$ (8), we obtain the consumption function.

$$
c(t)=\frac{W(t)}{\int_{t}^{T}\left(\left(\frac{R(s)}{R(t)}\right)^{1-\gamma} D_{r}(s-t)\right)^{1 / \gamma} d s}
$$

\footnotetext{
${ }^{16}$ See Appendix C for a rigorous derivation.
} 
which has MPC

$$
m(t)=\frac{1}{\int_{t}^{T}\left(\left(\frac{R(s)}{R(t)}\right)^{1-\gamma} D_{r}(s-t)\right)^{1 / \gamma} d s} .
$$

For comparison, the MPC for a time-consistent model with discounting function $D(t)$ is

$$
m_{D}(t)=\frac{1}{\int_{t}^{T}\left(\left(\frac{R(s)}{R(t)}\right)^{1-\gamma} \frac{D(s)}{D(t)}\right)^{1 / \gamma} d s} .
$$

This will be the same as (57) if

$$
D_{r}(s-t)=\frac{D_{r}(s)}{D_{r}(t)}
$$

As Strotz (1956) showed, this equality can only hold for all $(s, t) \in\left\{[0, T]^{2}: s \geq t\right\}$ if $D_{r}$ is exponential.

Like Eq. (58), Eq. (57) can be inverted to obtain the relative discount function that yields a given MPC function. However, because $s$ and $t$ cannot be multiplicatively separated in $D_{r}(s-t)$ like they can when the restriction (59) holds, stronger assumptions are needed to achieve a result analogous to Proposition 2 in Section 4.

Proposition 3 Let $m:[0, T] \rightarrow \mathbf{R}_{++}$satisfy (11) so it is the MPC for a proportional consumption rule. If $r(t)=r$ for all $t \in[0, T]$ or if $\gamma=1$, there exists $D_{r}:[0, T] \rightarrow \mathbf{R}_{++}$such that $D_{r}(0)=1$ and Eq. (57) holds.

Let $m(t)$ be the MPC for a proportional consumption rule. We can rewrite (57) as

$$
\int_{t}^{T}\left(\left(\frac{R(s)}{R(t)}\right)^{1-\gamma} D_{r}(s-t)\right)^{1 / \gamma} d s=\frac{1}{m(t)}
$$

Differentiating this by $t$,

$$
\frac{1}{m(t)} \frac{d \ln m(t)}{d t}=1+\int_{t}^{T} \frac{d}{d s}\left(D_{r}^{1 / \gamma}(s-t)\right)\left(\frac{R(s)}{R(t)}\right)^{\frac{1-\gamma}{\gamma}} d s+\frac{1-\gamma}{\gamma} r(t) \int_{t}^{T} D_{r}^{1 / \gamma}(s-t)\left(\frac{R(s)}{R(t)}\right)^{\frac{1-\gamma}{\gamma}} d s
$$

Integrating the second term by parts and reusing (60) to simplify the third term,

$$
\begin{aligned}
\frac{1}{m(t)} \frac{d \ln m(t)}{d t}= & 1+\left[D_{r}^{\frac{1}{\gamma}}(s-t)\left(\frac{R(s)}{R(t)}\right)^{\frac{1-\gamma}{\gamma}}\right]_{t}^{T} \\
& -\frac{1-\gamma}{\gamma} \int_{t}^{T} D_{r}^{1 / \gamma}(s-t) r(s)\left(\frac{R(s)}{R(t)}\right)^{\frac{1-\gamma}{\gamma}} d s+\frac{1-\gamma}{\gamma} \frac{r(t)}{m(t)}
\end{aligned}
$$

Thus

$$
\frac{1}{m(t)} \frac{d \ln m(t)}{d t}=D_{r}^{1 / \gamma}(T-t)\left(\frac{R(T)}{R(t)}\right)^{\frac{1-\gamma}{\gamma}}+\frac{1-\gamma}{\gamma}\left[\frac{r(t)}{m(t)}-\int_{t}^{T} D_{r}^{1 / \gamma}(s-t) r(s)\left(\frac{R(s)}{R(t)}\right)^{\frac{1-\gamma}{\gamma}} d s\right] .
$$

For the special cases where $r(t)=r$ or $\gamma=1$, the second term on the right of (61) cancels: ${ }^{17}$

$$
\frac{1}{m(t)} \frac{d \ln m(t)}{d t}=D_{r}^{1 / \gamma}(T-t) \exp \left(\frac{1-\gamma}{\gamma} r(T-t)\right)
$$

\footnotetext{
${ }^{17}$ For simplicity, we will write $r(t)=r$ in the following. With $\gamma=1, r(t)$ need not be constant. However, in that case, $r(t)$ cancels from the expressions that follow.
} 
Thus we can solve for $D_{r}(T-t)$.

$$
D_{r}(T-t)=\left(\frac{1}{m(t)} \frac{d \ln m(t)}{d t}\right)^{\gamma} \exp (-(1-\gamma) r(T-t))
$$

Let $s=T-t$, so $t=T-s$. Then we obtain

$$
D_{r}(s)=\left(\frac{1}{m(T-s)} \frac{d \ln m(T-s)}{d t}\right)^{\gamma} \exp (-(1-\gamma) r s)
$$

for the relative discounting function that yields the proportional consumption rule with MPC $m(t)$.

Since Propositions 2 and 3 establish that there is a 1-1 mapping between time-consistent discount functions $D(t)$ and the MPC function $m(t)$, and there is also a 1-1 mapping between relative discount functions $D_{r}(s)$ and the MPC function $m(t)$, there must also be a 1-1 mapping between the two types of discount functions. ${ }^{18}$

Corollary 4 In a steady state with constant interest rates or if utility is logarithmic, there is a one-to-one correspondence between the space of time-consistent discount functions $D(t):[0, T] \rightarrow \mathbf{R}_{+}$with $D(0)=1$ and the space of relative discounting functions $D_{r}(s):[0, T] \rightarrow \mathbf{R}_{+}$with $D_{r}(0)=1$ such that the same consumption behavior is realized in a time-consistent discounting model with $D(t)$ and in a time-inconsistent discounting model with the corresponding $D_{r}(s)$.

For both time-consistent and time-inconsistent discounting models, households are sufficiently rational so that lifetime wealth $W(t)$ is a sufficient statistic for their income path. The existence of a proportional consumption rule means that households can decompose their consumption-saving problem into the determination of their lifetime wealth and the determination of what fraction of their wealth they should consume at each age. The dichotomy between these two problems ensures that the MPC only depends on a household's current age and preferences, and not on the income path. Thus the mapping between the two types of discounting functions will be the same for any income path, though it does depend on the reigning interest rate (except when $\gamma=1$ ).

From a scientific perspective, the significance of Corollary 4 is that we will not be able to identify whether households maximize a time-consistent utility function like (45) or repeatedly reoptimize a time-inconsistent utility function like (54) if we only have access to panel data on household consumption. The two types of preference models can only be distinguished empirically if we can elicit a household's plans for future consumption at various points in the lifecycle. If the household repeatedly reoptimizes a time-inconsistent utility function, its plan for how much it will consume at some future age $t$ can change as the household progresses through time to age $t$. In constrast, if the household maximizes a time-consistent utility function, its plan should remain unchanged over the course of the lifecycle.

\footnotetext{
${ }^{18}$ This corollary does not imply that the equivalence between a time-consistent discount function and a relative discounting function preserves the monotonicity properties of the discount functions. A model with a strictly decreasing time-consistent discount function might have a relative discounting function that is not decreasing, or vice versa. In a finite-horizon model, there is no technical necessity of having a decreasing discount function. Most economists intuitively believe that discount functions ought to be decreasing. In practice we will usually start off with one representation of the discount function that we know how to interpret and which may satisfy monotonicity. The corollary shows there is a mathematically equivalent representation of the other type that may be useful for some purposes, but we do not have to abandon the original, monotonic representation when interpreting these preferences.
} 


\section{Calibration Exercises}

I now apply Corollary 4 to some common discounting models in the literature, calibrated to match important macroeconomic observables. Where economists normally employ a relative discounting model, we obtain the equivalent time-consistent model, and vice versa.

\subsection{Computing the Equivalent Time-Consistent Discount Function}

First, let us compare the equivalent time-consistent discount functions for the exponential, hyperbolic, and myopic discounting models described in Section 3. I have calibrated an overlapping-generations model where households live with certainty from age 25 to age 80 . Household income $y(t)$ follows Gourinchas and Parker (2002) until households retire at age 65 . The interest rate $r=3.4 \%{ }^{19}$

One issue that has to be addressed when comparing across discounting models is that discounting parameters do not have the same economic interpretation. The same value of the "discount rate" in two different discounting models may yield different levels of impatience. Here I control for patience by calibrating the three models so aggregate saving

$$
K=\int_{0}^{T} k(t) d t
$$

is the same across models. ${ }^{20}$ With $\log$ utility, I set $\rho=0.04$ in the exponential model, $\eta=0.063$ in the hyperbolic model, and $H=19$ years (with $\rho=0$ ) in the myopic model. ${ }^{21}$

The resulting consumption profiles are plotted in Fig. 1. The exponential model produces a smooth, almost linear consumption profile that slopes downward because $\rho>r$. The hyperbolic model produces a hump-shaped consumption profile as in Park (2012). The myopic model gives a piecewise smooth profile. Prior to age 61, the household's time horizon is shorter than its lifespan, so it has an effective discount rate of $\rho_{\text {eff }}=\frac{1}{H}=0.052>r$, and consumption declines steeply. After age 61, the household does not discount future consumption, so consumption rises even more steeply since $r>0$.

The corresponding asset demand profiles are plotted in Fig. 2. The area under each curve is the same by construction. All three models lead the household to initially borrow and then build up saving to fund consumption during retirement. The hyperbolic model actually has the household borrow the least and start saving earliest, although it accumulates less saving prior to retirement. The myopic model borrows the most, but also accumulates the most saving at retirement since it consumes so little in the years prior to the realization that the household will soon reach its end.

While the hyperbolic and myopic models are usually viewed as less rational than the exponential model, all three consumption profiles can be obtained from a time-consistent discounting model that is unquestionably rational. The equivalent discount function $D(t)$ for the three models is given in Fig. 3. The myopic model has a piecewise exponential function with the discount rate in each piece equal to the effective discount rates computed above. Early in life, the hyperbolic model puts more weight on consumption than the other two models whereas the myopic model puts less weight on consumption until about age 72 when the three discount functions intersect. Thereafter the ordering of the three discount functions reverses. The relative

\footnotetext{
${ }^{19}$ This is chosen to produce $K / Y=2.5$ and $C / Y=0.75$ with a Cobb-Douglas production technology that has a share of capital of $1 / 3$ and depreciation rate of $10 \%$ in an overlapping-generations model with labor productivity endowment $e(t)=y(t) / w$, where the wage $w$ is determined in equilibrium.

${ }^{20} \mathrm{~A}$ more common method of controlling for patience is to equalize $\int_{t}^{T} D_{r}\left(t^{\prime}\right) d t^{\prime}$ across models, but this can only be done for one $t$. Equalizing aggregate saving controls for patience across the lifecycle. Moreover, aggregate saving has the advantage that it is observable.

${ }^{21} \mathrm{With}$ these parameters, aggregate saving equals the capital stock necessary to achieve $r=3.4 \%$ in equilibrium.
} 


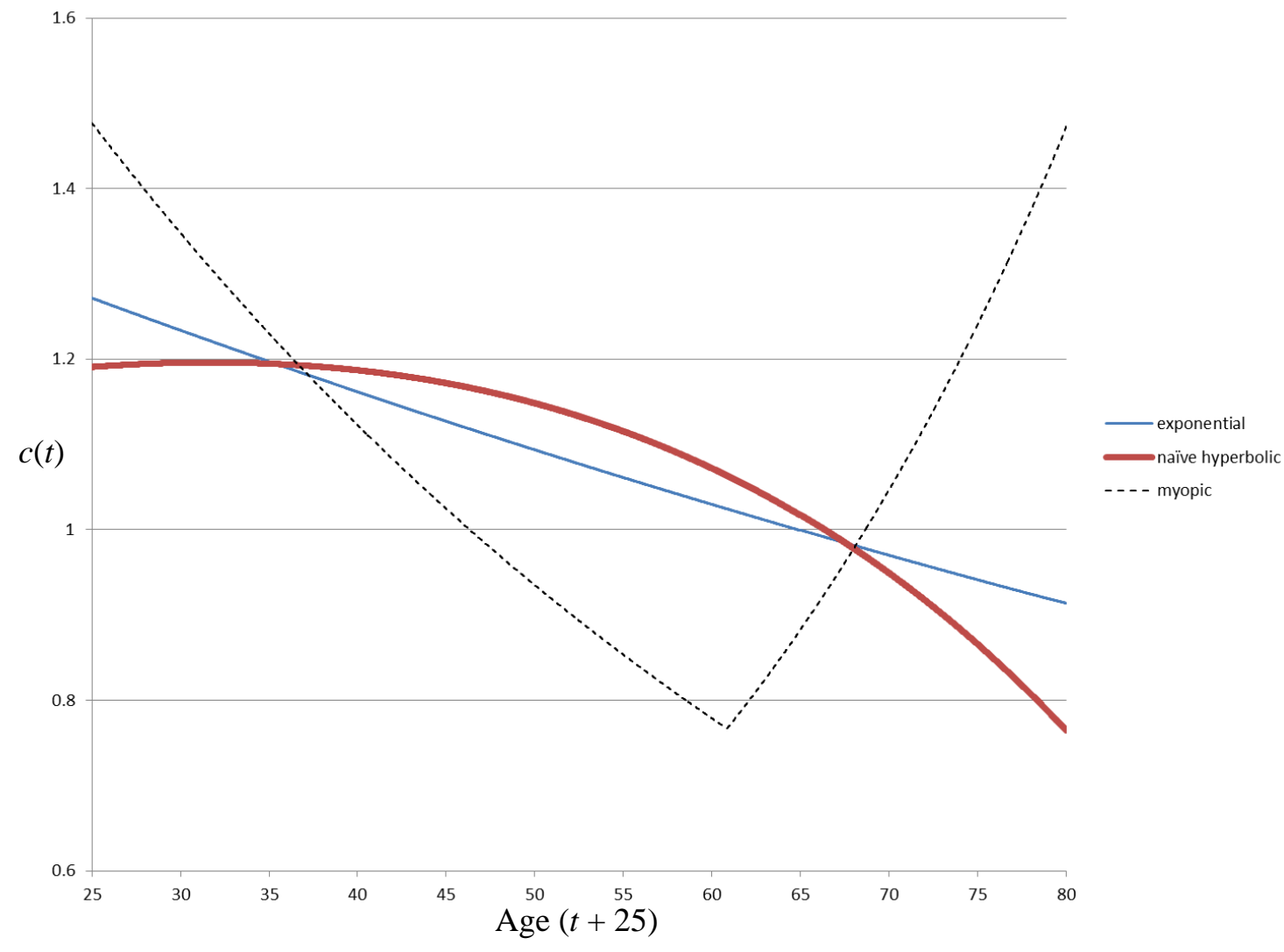

Figure 1: Lifecycle consumption profiles for the exponential, hyperbolic, and myopic discounting models calibrated to produce the same aggregate saving. 


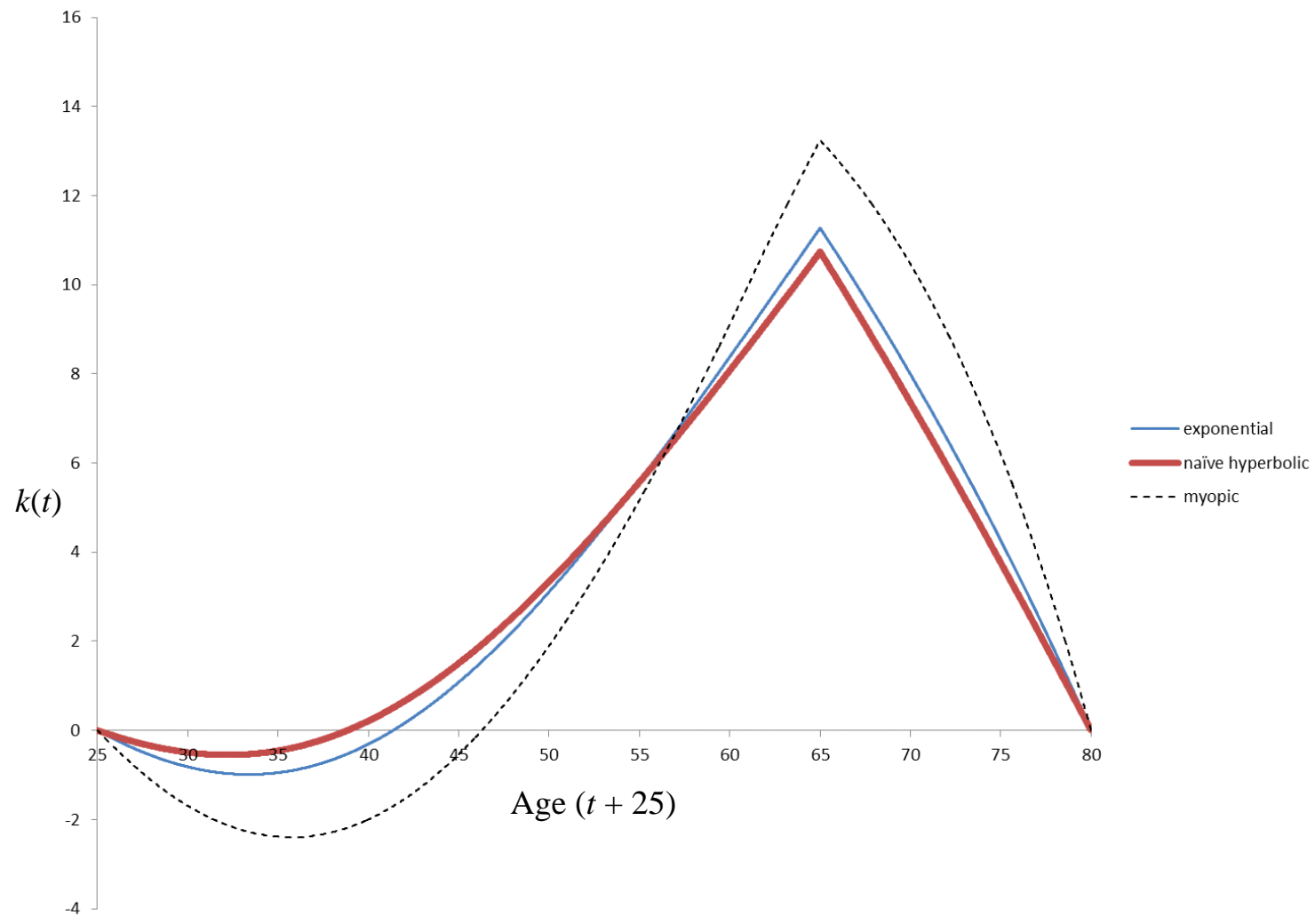

Figure 2: Lifecycle asset demand profiles $k(t)$ for the exponential, hyperbolic, and myopic discounting models calibrated to produce the same aggregate saving. 
weight

$$
\frac{D(t)}{D_{\exp }(t)}-1
$$

of the hyperbolic and myopic discount functions to the exponential is plotted in Fig. 4. The hyperbolic model puts a maximum of $12.5 \%$ more weight than the exponential model on utility in the early fifties. At the end of life, it puts $10 \%$ less weight on utility than the exponential model. In contrast, the myopic model puts a minimum of $40 \%$ less weight than the exponential model on utility in the $60 \mathrm{~s}$. At the end of life, it puts $40 \%$ more weight on utility.

The exponential discounting model assumes a constant decay rate for the discount function. However, if preferences are dictated by biological forces, an idea discussed at greater length in the conclusion, then it is plausible that human beings would evolve to put more value on consumption during the first half of adulthood when they are likely to be producing and raising children. Purely hyperbolic discounting may put too much weight on consumption during the middle ages and not enough during the childbearing years. Nevertheless if such biological factors do play a role in driving preferences, the hyperbolic model will better approximate the true discounting function than the exponential model.

\subsection{Computing the Equivalent Relative Discount Function}

Another common discounting model employed in the literature ${ }^{22}$ is the standard exponential discounting model augmented by mortality risk, which is time-consistent:

$$
D(t)=\exp (-\rho t) Q(t)
$$

I calibrate this model with a discount rate of $\rho=0.04$ and a survivor function $Q(t)$ taken from U.S. data (Bullard and Feigenbaum (2007)). The equivalent relative discount function is plotted in Fig. 5. Note that the resulting relative discount function $D_{r}(t)$ is not monotonic decreasing, a property that is not required by our definition of a relative discount function. For very small $t$, the reported $D_{r}(t)$ function behaves quite weirdly, starting at 1 , dropping down to 0.7 and then returning to 0.9 over delays between 0 and a month. This is likely due to approximation error. The behavior of $D_{r}$ at short delays is driven by the behavior of the MPC function at the end of life, where $m(t)$ is divergent.

I also plot $D_{r}^{h y p}(t ; 0.12)$, which is the closest fit to $D_{r}(t)$ in the hyperbolic class of relative discount functions, and $D_{r}^{\exp }(t ; 0.45)$ in the exponential class. Although the relative discount model is not well represented by either model, the hyperbolic model provides a better fit than the exponential model. ${ }^{23}$ Thus another explanation for why human beings might exhibit non-exponential discounting is that this is a convenient way to incorporate mortality risk in the discount function.

\section{Social Security}

Imrohoroglu, Imrohoroglu, and Joines (2003) have shown that Social Security cannot improve the welfare of hyperbolic discounters in partial equilibrium. Caliendo (2011) showed this more generally for any timeinconsistent preferences of the form (54). His Generalized IIJ Impossibility Theorem can be seen immediately

\footnotetext{
${ }^{22}$ See, for example, Huang, Milevsky, and Salisbury (2012); Richard (1975); or Yaari (1965).

${ }^{23}$ The mean squared error for the hyperbolic fit is 0.020 while it is 0.045 for the exponential fit.
} 


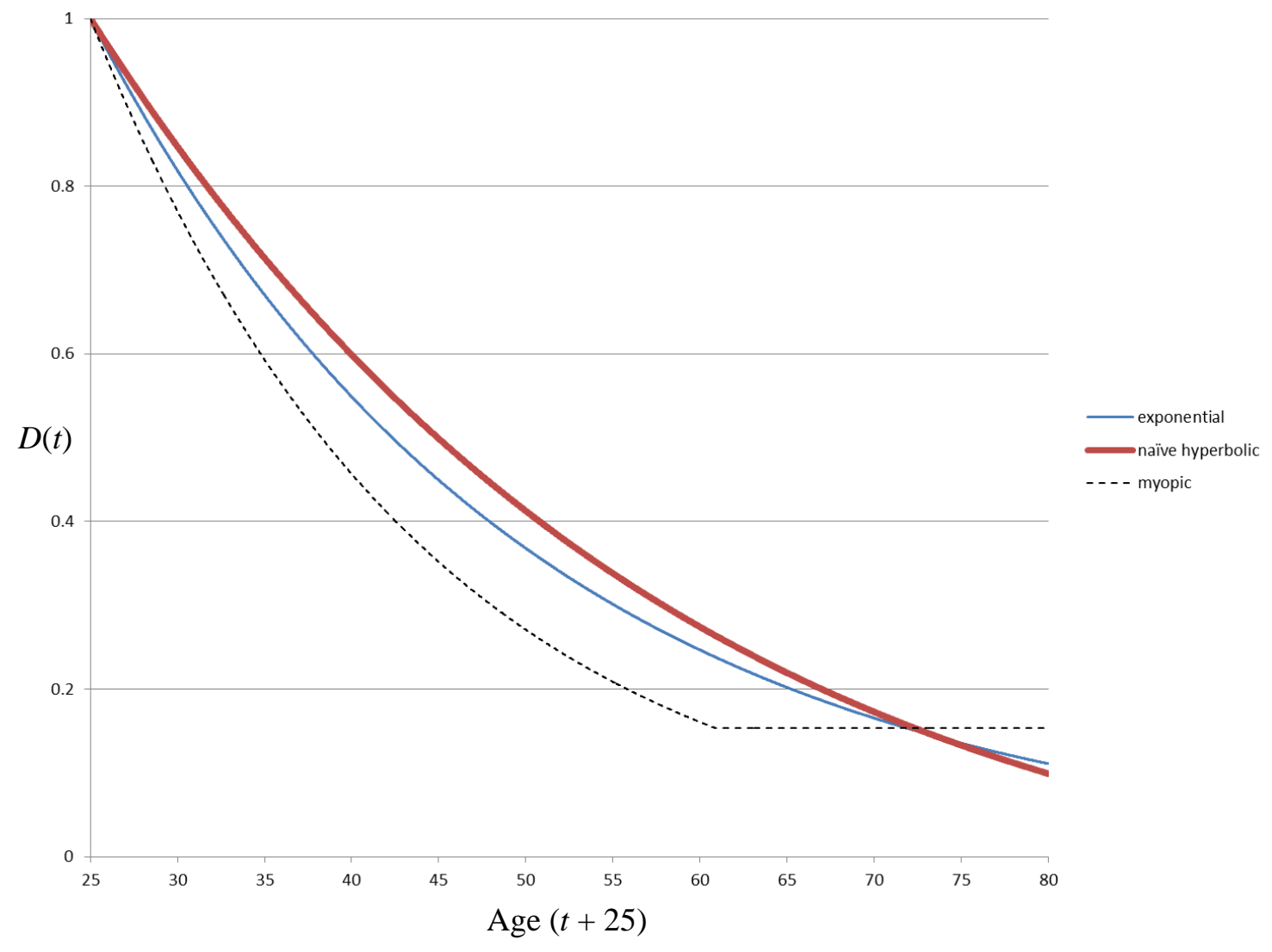

Figure 3: Time-consistent discount functions $D(t)$ for the exponential, hyperbolic, and myopic discounting models calibrated to produce the same aggregate saving. 


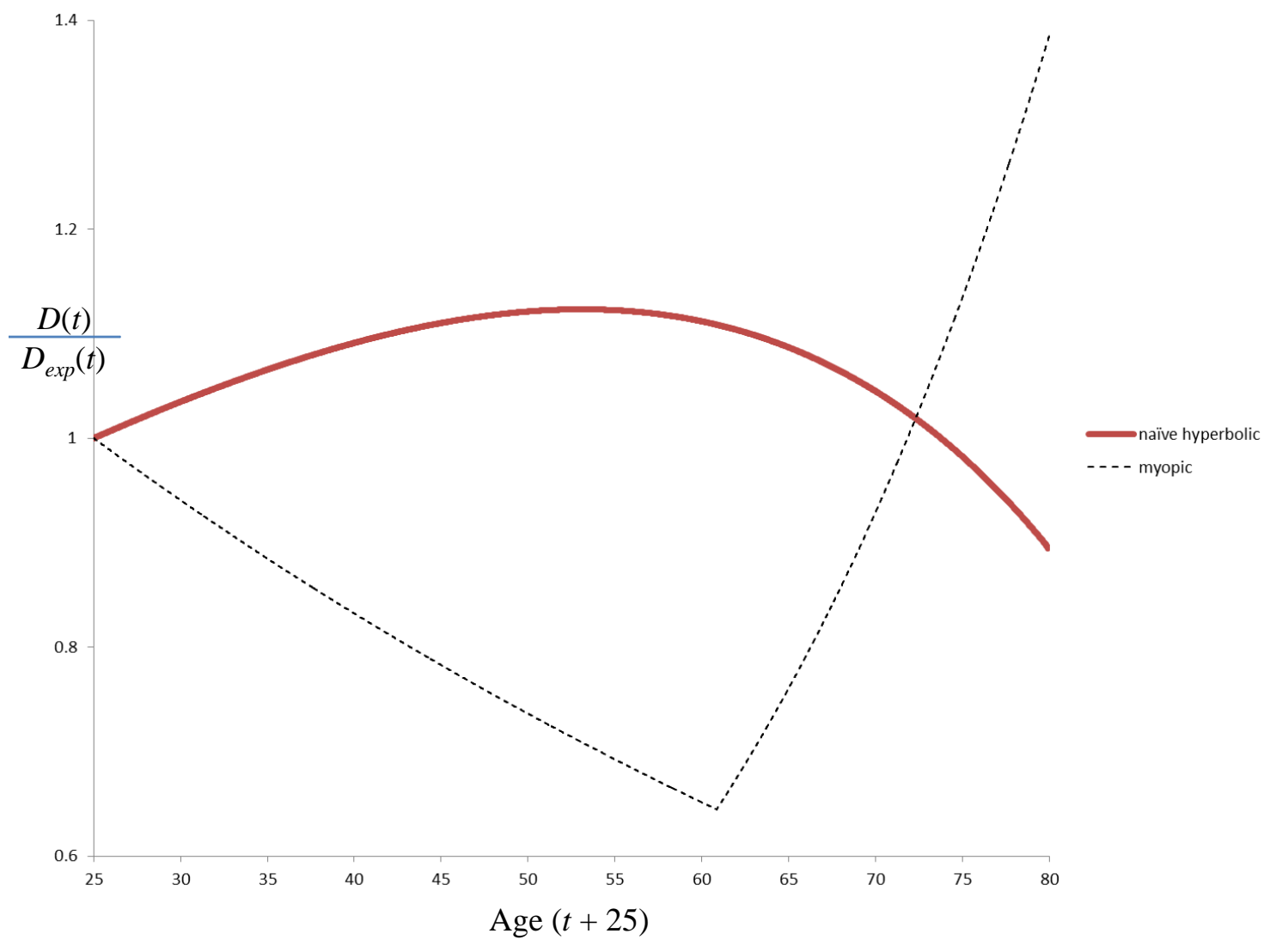

Figure 4: Discount functions $D(t)$ for the hyperbolic and myopic discounting models relative to the exponential model. 


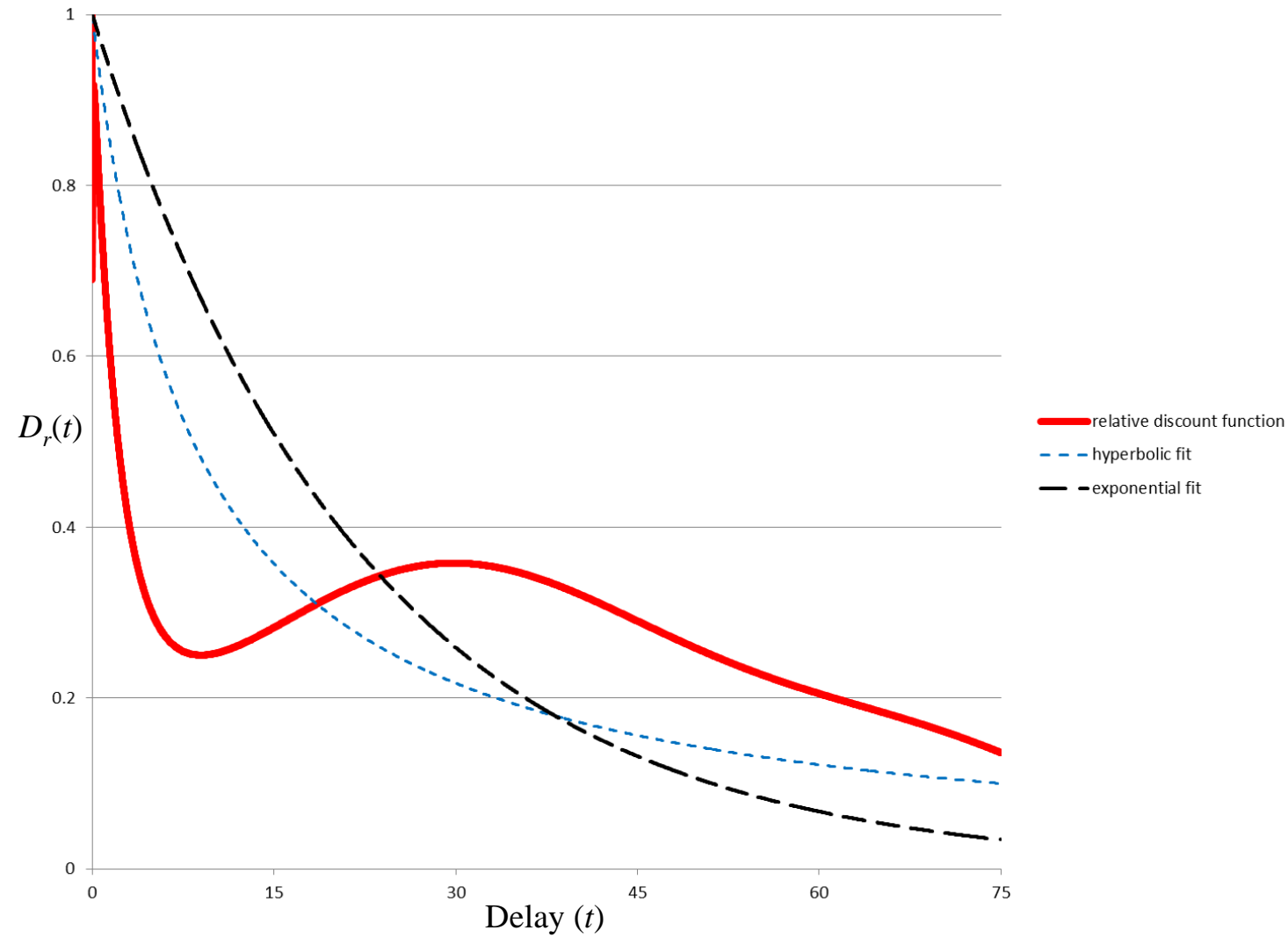

Figure 5: The relative discount function $D_{r}(t)$ equivalent to a time-consistent discount function $Q(t) \exp (-r t)$. Fits to exponential and hyperbolic discounting functions are also plotted. 
if we use the equivalent time-consistent discount function to evaluate welfare. In the present context, we can analyze the effect of changing $y(t)$ without changing the path of interest rates $r(t) .{ }^{24}$

From Eq. (21), we see that consumption at any point in the lifecycle will be proportional to the present value of income $\int_{0}^{T} \frac{y(s)}{R(s)} d s$. If $r(t)$ is fixed, the proportionality factor $m(t) \frac{R(t)}{M(t)}$ will be exogenous, and the household will be objectively worse off if a policy reduces the present value of income. With monotone, timeconsistent preferences, lifetime utility must then also be reduced. Combining (45) and (21), we compute lifetime welfare with the time-consistent representation of utility:

$$
U=\int_{0}^{T} D(t) u\left(m(t) \frac{R(t)}{M(t)} \int_{0}^{T} \frac{y(s)}{R(s)} d s\right) d t
$$

For $\gamma \neq 1$,

$$
U=u\left(\int_{0}^{T} \frac{y(s)}{R(s)} d s\right) \int_{0}^{T} D(t)\left(m(t) \frac{R(t)}{M(t)}\right)^{1-\gamma} d t
$$

while for the case of log utility

$$
U=\ln \left(\int_{0}^{T} \frac{y(s)}{R(s)} d s\right) \int_{0}^{T} D(t) d t+\int_{0}^{T} D(t) \ln \left(m(t) \frac{R(t)}{M(t)}\right) d t .
$$

In either case, lifetime welfare is a strictly increasing function of the present value of income $\int_{0}^{T} \frac{y(s)}{R(s)} d s$. Thus the effect of any policy on welfare in partial equilibrium is solely determined by how this policy affects the household's wealth.

In partial equilibrium without binding borrowing constraints, Social Security cannot improve the welfare of naive time-inconsistent individuals if it reduces the present value of lifetime income, regardless of the shape of the discount function. In equivalent terms, Social Security must reduce welfare if its internal rate of return is less than the interest rate. I should stress, though, that this conclusion only applies to welfare as defined by the equivalent time-consistent discount function, which I am presuming must drive the evolution of the household's behavior. This does not imply that Social Security cannot make households feel better off as they perceive their welfare if in fact they have time-inconsistent preferences. ${ }^{25}$

This result is a consequence of the fact that agents in these models still follow consumption rules proportional to current lifetime wealth, which households are assumed to value correctly. If households have irrational expectations about their future income, as in Caliendo and Aadland (2007) or Park (2009), then Social Security can make a difference. Findley and Caliendo (2008) have demonstrated that when households are not just myopic in their preferences but also cannot see that they will earn income beyond their current time horizon, then Social Security can raise welfare. In such models, the reason why households do not save enough for retirement is not because they undervalue retirement consumption during their working life; it is because they do not expect to retire.

Another argument for how Social Security might help households is that it may act as a commitment device that prevents households with time-inconsistent preferences from deviating from their original time-0 plan. In fact Guo and Caliendo (2012) have shown that a self-financing transfer-payment mechanism that keeps households from deviating from their original time-0 plan must involve time-inconsistent announcements about what benefits households can expect from the government and consequently looks nothing like

\footnotetext{
${ }^{24}$ We cannot use the equivalent time-consistent model for welfare comparison in general equilibrium, except in the case of log utility, because a change in Social Security will change the interest rate and therefore change the mapping.

${ }^{25}$ Similar objections can also be made regarding the interpretation of the preexisting IIJ theorems. IIJ (2003) and Caliendo (2011) draw their conclusions by showing that consumption at every point in the lifecycle will be reduced if Social Security has a negative net present value.
} 
existing Social Security systems. But even if Social Security could work as a commitment device, we can use the equivalent time-consistent discounting function to ask whether it is a good idea for the government to provide this commitment device. Both the original plan and the realized plan will be feasible. Since a household with the time-consistent discounting function is completely rational, the realized plan will be chosen over the original plan precisely because the realized plan delivers higher utility. Thus there is no time-consistent value in helping households with time-inconsistent preferences commit to their original plan, and this should neither be helped nor encouraged.

\section{Concluding Remarks and Evolutionary Interpretation of Equiv- alence Relation}

Suppose that households value their consumption flow using a constant relative risk aversion utility function. If households allocate consumption to maximize the integral of this utility flow over the lifecycle, weighted by a time-consistent discount function, they will adopt a proportional consumption rule in which their consumption at every instant is some age-dependent fraction of their accumulated wealth at that age. We call this fraction the marginal propensity to consume. If, instead, at every instant households maximize the integral of the utility flow over their remaining life span, weighted by a time-inconsistent relative discount function, they will also adopt a proportional consumption rule. Only in the case of an exponential discount function will the resulting rule be the same for both classes of models if the functional form of the discount function is the same. Nevertheless, I have shown that in a steady state with constant interest rates, given a specification of how the MPC varies over the lifecycle, we can obtain both a time-consistent discount function and a relative discount function that generate a given marginal propensity to consume function. Consequently, there is a one-to-one correspondence between time-consistent discounting models and timeinconsistent discounting models: a time-consistent discounting model and its corresponding time-inconsistent model will both produce the same consumption behavior.

To continue the quantum mechanical analogy from the introduction, there is a duality between timeconsistent and time-inconsistent discounting models. We can exploit this duality to better understand behavior that, at least at first glance, seems like it can only come from one class of model and not the other, as for example occurs when experimental economists observe preference reversals.

If we accept the premise that preferences over consumption have evolved to optimally satisfy biological imperatives then natural selection requires a time-consistent representation of preferences to work upon since the time scale of natural selection is much longer than one generation. Under this view, the timeconsistent discount function ought to measure the relative importance of consumption at different ages for the perpetuation of the species. However, while a time-consistent representation is necessary to determine the optimal consumption rule, it is not necessary to implement a proportional consumption rule. A brain with finite computing power may find it easier to employ a relative discounting function when making consumption-saving decisions since the relative discounting function that yields the optimal consumption rule will not change over the lifecycle. The two representations will only coincide for the special case when the time-consistent discounting function is exponential, which is a subset of measure zero of the space of possible discounting functions. Thus it will almost certainly be the case that the optimal consumption rule can be derived from a time-inconsistent relative discounting function.

I have shown that equivalent time-consistent discount functions for hyperbolic and similar relative discount functions put more weight on consumption during and prior to the child-bearing years. Mortality risk will naturally cause a person to put more weight on consumption early in life. From a biological perspective, more weight should also be put on consumption during these years if preferences are determined so as to 
maximize the size of one's progeny. The health of a person after his kids are grown has very little impact on the perpetuation of his line. Consumption prior to reproduction is important to ensure healthy children, and consumption, both of the parent and child, while the children are growing will also be important. Thus consumption behavior consistent with hyperbolic-like discounting is also consistent with biological theory.

Since the two representations are equivalent for the purposes of solving macroeconomic overlappinggenerations models, we suggest that the most relevant representation be applied depending on the context. When doing welfare analysis, one ought to use the time-consistent discounting model that must presumably determine why the observed consumption rule is optimal. ${ }^{26}$ When doing experiments that delve into precisely how consumption choices are made, one should use the discounting model that best matches how people empirically make these choices, which, based on existing data, is going to be the relative discounting model. The upshot of all this is that time-inconsistent preferences that arise from a relative discounting model are an innocuous deviation from our standard notion of rational behavior. If, on the other hand, preference reversals are happening because the brain is only solving an approximate version of the household's problem (Rubinstein (1988, 2003), Leland (2002)), that is a situation not covered by the models in this paper and welfare consequences may be more severe.

The findings of this paper emphasize the need for a reliable panel data set on consumption. It has been more than 50 years since Friedman (1957) wrote A Theory of the Consumption Function. After five decades, we still have only an imprecise understanding of how people apportion their income between consumption and savings. The state of the art today is to assume a family of discounting models, usually exponential or hyperbolic, and estimate which parameters fit best to consumption data (Blow, Browning, and Crawford (2014)). ${ }^{27}$ If we knew more precisely what consumption rule people actually obey, we could nonparametrically back out preferences consistent with this consumption rule, and many of our debates about preferences would be resolved.

\section{A Properties of Standard Discounting Models}

\section{A.1 Exponential}

The Hamiltonian for the exponential problem (22) is

$$
H_{\exp }=\exp (-\rho t) u(c(t))+\lambda(t)[y(t)+r(t) k(t)-c(t)]
$$

which yields the equations of motion

$$
\begin{gathered}
\frac{\partial H_{\exp }}{\partial c(t)}=\exp (-\rho t) u^{\prime}(c(t))-\lambda(t)=0 \\
\frac{\partial H_{\exp }}{\partial k(t)}=r(t) \lambda(t)=-\frac{d \lambda(t)}{d t} .
\end{gathered}
$$

The $c$ equation (68) determines $\lambda(t)$ as a function of $c(t)$

$$
\lambda(t)=\exp (-\rho t) u^{\prime}(c(t))
$$

\footnotetext{
${ }^{26}$ We are assuming here that the utility function maximized by natural selection is also the utility function that the individual would prefer to maximize. Philosophically, this is a rather shaky proposition, equivalent to assuming that people have no free will independent of their biologically determined fate. As a practical matter though, if we abandon the basic principle of revealed preference that we can deduce an individual's utility function from his behavior, then we are in Austrian territory.

${ }^{27}$ We can compute both the time-consistent and the observationally equivalent time-inconsistent discount function that best fit household consumption data. However, we cannot distinguish which of these two discount functions the household actually employs in its utility function.
} 
while the $k$ equation (69) determines the equation of motion for $\lambda(t)$ :

$$
\frac{d \ln \lambda(t)}{d t}=-r(t)
$$

which has the solution

$$
\lambda(t)=\frac{\lambda(0)}{R(t)}
$$

Solving (70) for $c(t)$

$$
c(t)=\left(u^{\prime}\right)^{-1}\left(\frac{\lambda(0) \exp (\rho t)}{R(t)}\right)
$$

and defining

$$
c(0)=\left(u^{\prime}\right)^{-1}(\lambda(0))
$$

we obtain the lifecycle consumption profile

$$
c(t)=\left(u^{\prime}\right)^{-1}\left(\frac{u^{\prime}(c(0)) \exp (\rho t)}{R(t)}\right) .
$$

Solving (73) for $c(0)$, and inserting that result back into (73) evaluated at $s \in[t, T]$, we find

$$
c(s)=\left(u^{\prime}\right)^{-1}\left(u^{\prime}(c(t)) \exp (\rho(s-t)) \frac{R(t)}{R(s)}\right) .
$$

Substituting this into the lifetime budget constraint (8), we obtain the exponential MPC

$$
m_{\exp }(t ; \rho)=\frac{1}{\int_{t}^{T}\left(\frac{R(t)}{R(s)}\right)^{\frac{\gamma-1}{\gamma}} \exp \left(-\frac{\rho}{\gamma}(s-t)\right) d s} .
$$

Note that $m_{\exp }(t ; \rho)$ satisfies (11), for

$$
\begin{aligned}
\lim _{t \rightarrow T} m_{\exp }(t ; \rho)(T-t) & =\lim _{t \rightarrow T} \frac{T-t}{\int_{t}^{T}\left(\frac{R(t)}{R(s)}\right)^{\frac{\gamma-1}{\gamma}} \exp \left(-\frac{\rho}{\gamma}(s-t)\right) d s} \\
& =\lim _{t \rightarrow T} \frac{-1}{\int_{t}^{T} \frac{\partial}{\partial t}\left[\left(\frac{R(t)}{R(s)}\right)^{\frac{\gamma-1}{\gamma}} \exp \left(-\frac{\rho}{\gamma}(s-t)\right)\right] d s-\left(\frac{R(t)}{R(t)}\right)^{\frac{\gamma-1}{\gamma}} \exp \left(-\frac{\rho}{\gamma}(t-t)\right)}=1
\end{aligned}
$$

by l'Hôpital's Rule.

\section{A.2 Naive Hyperbolic}

The Hamiltonian for the hyperbolic problem (31) at age $t$ is

$$
H_{\text {hyp }}(t)=\frac{u(c(s ; t) ; \gamma)}{1+\eta(s-t)}+\lambda(s)[y(s)+r(s) k(s ; t)-c(s ; t)] .
$$

Hamilton's equations are

$$
\frac{\partial H_{h y p}(t)}{\partial c(s ; t)}=\frac{c(s ; t)^{-\gamma}}{1+\eta(s-t)}-\lambda(s)=0
$$




$$
\frac{d \lambda(s)}{d s}=-\frac{\partial H_{h y p}(t)}{\partial k(s ; t)}=-\lambda(s) r(s) .
$$

The costate variable has the solution

$$
\lambda(s)=\frac{\lambda(t) R(t)}{R(s)}
$$

From Eq. (75), planned consumption is

$$
c(s ; t)=[\lambda(s)(1+\eta(s-t))]^{-1 / \gamma} .
$$

If we define

$$
c(t)=\lambda(t)^{-1 / \gamma}
$$

we can rewrite $(78)$ as

$$
c(s ; t)=\left(\frac{R(s)}{R(t)(1+\eta(s-t))}\right)^{1 / \gamma} c(t) .
$$

Substituting this into the imagined budget constraint (36), we obtain the hyperbolic marginal propensity to consume

$$
m_{\text {hyp }}(t ; \eta)=\frac{1}{\int_{t}^{T}\left(\frac{R(s)}{R(t)}\right)^{\frac{1-\gamma}{\gamma}}[1+\eta(s-t)]^{-1 / \gamma} d s} .
$$

Since

$$
\begin{aligned}
\lim _{t \rightarrow T} m_{\text {hyp }}(t ; \eta)(T-t) & =\lim _{t \rightarrow T} \frac{T-t}{\int_{t}^{T}\left(\frac{R(s)}{R(t)}\right)^{\frac{1-\gamma}{\gamma}}[1+\eta(s-t)]^{-1 / \gamma} d s} \\
& =\lim _{t \rightarrow T} \frac{-1}{\int_{t}^{T} \frac{\partial}{\partial t}\left[\left(\frac{R(s)}{R(t)}\right)^{\frac{1-\gamma}{\gamma}}[1+\eta(s-t)]^{-1 / \gamma}\right] d s-\left(\frac{R(t)}{R(t)}\right)^{\frac{1-\gamma}{\gamma}}[1+\eta(t-t)]^{-1 / \gamma}}=1,
\end{aligned}
$$

the naive hyperbolic MPC satisfies the condition (11)

\section{A.3 Myopic}

The Hamiltonian at age $t$ will be

$$
\begin{gathered}
H_{\text {myo }}(t)=\exp (-\rho(s-t)) u(c(s ; t))+\lambda(s)[y(s)+r(s) k(s ; t)-c(s ; t)] . \\
\frac{\partial H_{m y o}}{\partial c(s ; t)}=\exp (-\rho(s-t)) u^{\prime}(c(s ; t))=0 \\
\frac{d \lambda(s)}{d t}=-\frac{\partial H_{m y o}}{\partial k(s ; t)}=-r(s) \lambda(s) .
\end{gathered}
$$

These equations of motion are the same as in Section 3.1 except they are only applicable for $s \in[t, \min \{t+$ $H, T\}]$. Thus (23) is applicable for $s<\min \{t+H, T\}$, and the planned lifecycle consumption profile at age $t$ is

$$
c(s ; t)=\left\{\begin{array}{cc}
c(t)\left(\frac{R(s)}{R(t)}\right)^{1 / \gamma} \exp \left(-\frac{\rho}{\gamma}(s-t)\right) & s \in[t, \min \{t+H, T\}] \\
0 & s \in(t+H, T]
\end{array} .\right.
$$


Inserting this into the imagined lifetime budget constraint (36), we obtain

$$
m_{\text {myo }}(t ; \rho, H)=\frac{1}{\int_{t}^{\min \{t+H, T\}}\left(\frac{R(s)}{R(t)}\right)^{\frac{1-\gamma}{\gamma}} \exp \left(-\frac{\rho}{\gamma}(s-t)\right) d s} .
$$

Note that

$$
\begin{aligned}
\lim _{t \rightarrow T} m_{m y o}(t ; \rho, H)(T-t) & =\lim _{t \rightarrow T} \frac{T-t}{\int_{t}^{\min \{t+H, T\}}\left(\frac{R(s)}{R(t)}\right)^{\frac{1-\gamma}{\gamma}} \exp \left(-\frac{\rho}{\gamma}(s-t)\right) d s} \\
& =\lim _{t \rightarrow T} \frac{-1}{-\left(\frac{R(t)}{R(t)}\right)^{\frac{1-\gamma}{\gamma}} \exp \left(-\frac{\rho}{\gamma}(t-t)\right)+\int_{t}^{T} \frac{\partial}{\partial t}\left\{\left(\frac{R(s)}{R(t)}\right)^{\frac{1-\gamma}{\gamma}} \exp \left(-\frac{\rho}{\gamma}(s-t)\right)\right\} d s}=1 .
\end{aligned}
$$

\section{B Properties of General Discounting Models}

The Hamiltonian for the problem (45) with discount function $D$ is

$$
\begin{gathered}
H_{D}=D(t) u(c(t))+\lambda(t)[y(t)+r(t) k(t)-c(t)] \\
\frac{\partial H_{D}}{\partial c(t)}=D(t) u^{\prime}(c(t))-\lambda(t)=0 \\
\frac{d \lambda(t)}{d t}=-\frac{\partial H_{D}}{\partial k(t)}=-r(t) \lambda(t) .
\end{gathered}
$$

Given $\lambda(t)$, the solution to (83) is again (77) while the solution to (82) is

$$
c(t)=\left(\frac{\lambda(t)}{D(t)}\right)^{-1 / \gamma} .
$$

The MPC for the general discounting function is

$$
m(t)=\frac{1}{\int_{t}^{T}\left(\frac{D(s)}{D(t)}\left(\frac{R(s)}{R(t)}\right)^{1-\gamma}\right)^{1 / \gamma} d s}
$$

which satisfies the condition (11):

$$
\begin{aligned}
\lim _{t \rightarrow T} m(t)(T-t) & =\lim _{t \rightarrow T} \frac{T-t}{\int_{t}^{T}\left(\frac{D(s)}{D(t)}\left(\frac{R(s)}{R(t)}\right)^{1-\gamma}\right)^{1 / \gamma} d s} \\
& =\lim _{t \rightarrow T} \frac{-1}{\int_{t}^{T} \frac{\partial}{\partial t}\left(\frac{D(s)}{D(t)}\left(\frac{R(s)}{R(t)}\right)^{1-\gamma}\right)^{1 / \gamma} d s-\left(\frac{D(t)}{D(t)}\left(\frac{R(t)}{R(t)}\right)^{1-\gamma}\right)^{1 / \gamma}}=1
\end{aligned}
$$




\section{Properties of Relative Discounting Models}

The Hamiltonian for the problem (54) at age $t$ is

$$
H_{r}(t)=D_{r}(s-t) u(c(s))+\lambda(s)[y(s)+r(t) k(s)-c(s)] .
$$

Hamilton's equations are

$$
\begin{gathered}
\frac{\partial H_{r}}{\partial c(s)}=D_{r}(s-t) c(s)^{-\gamma}-\lambda(s)=0 \\
\frac{d \lambda(s)}{d s}=-\frac{\partial H_{r}}{\partial k(s)}=-r(s) \lambda(s) .
\end{gathered}
$$

The solution to (86)-(87) is

$$
c(s)=c(t)\left(\frac{R(s)}{R(t)} D_{r}(s-t)\right)^{1 / \gamma}
$$

Note that the resulting MPC,

$$
m(t)=\frac{1}{\int_{t}^{T}\left(\left(\frac{R(s)}{R(t)}\right)^{1-\gamma} D_{r}(s-t)\right)^{1 / \gamma} d s},
$$

satisfies (11):

$$
\begin{aligned}
\lim _{t \rightarrow T} m(t)(T-t) & =\lim _{t \rightarrow T} \frac{T-t}{\int_{t}^{T}\left(\left(\frac{R(s)}{R(t)}\right)^{1-\gamma} D_{r}(s-t)\right)^{1 / \gamma} d s} \\
& =\lim _{t \rightarrow T} \frac{-1}{-1+\int_{t}^{T} \frac{\partial}{\partial t}\left(\left(\left(\frac{R(s)}{R(t)}\right)^{1-\gamma} D_{r}(s-t)\right)^{1 / \gamma}\right) d s}=1 .
\end{aligned}
$$

Since

$$
\lim _{t \rightarrow T} m(t)(T-t)=1
$$

for $t \approx T$,

$$
\begin{gathered}
m(t) \approx \frac{1}{T-t} . \\
\frac{d \ln m_{r}(t)}{d t} \approx \frac{d}{d t}(-\ln (T-t))=\frac{1}{T-t}
\end{gathered}
$$

we also have

$$
\begin{aligned}
\lim _{s \rightarrow 0} D_{r}(s) & =\lim _{s \rightarrow 0}\left(\frac{1}{m(T-s)} \frac{d \ln m(T-s)}{d t}\right)^{\gamma} \exp (-(1-\gamma) r s) \\
& =\lim _{s \rightarrow 0}(T-(T-s)) \frac{1}{T-(T-s)}=1 .
\end{aligned}
$$




\section{References}

[1] Barro, Robert J., (1999), "Ramsey Meets Laibson in the Neoclassical Growth Model," Quarterly Journal of Economics 114: 1125-1152.

[2] Blow, Laura, Martin Browning, and Ian Crawford, (2014), "Never Mind the Hyperbolics: Nonparametric Analysis of Time-Inconsistent Preferences," Working Paper.

[3] Bullard, James and James A. Feigenbaum, (2007), "A Leisurely Reading of Lifecycle Consumption Data," Journal of Monetary Economics 54: 2305-2320.

[4] Caliendo, Frank N., (2011), "Time-Inconsistent Preferences and Social Security: Revisited in Continuous Time," Journal of Economic Dynamics and Control 35: 668-675.

[5] Caliendo, Frank and David Aadland, (2007), "Short-Term Planning and the Life-Cycle Consumption Puzzle," Journal of Economic Dynamics and Control 31: 1392-1415.

[6] Caliendo, Frank N. and Scott Findley, (2013), "Discounting Functions and Intertemporal Choice," Working Paper.

[7] Feigenbaum, James, Frank Caliendo, and Emin Gahramanov, (2011), "Optimal Irrational Behavior," Journal of Economic Behavior and Organization 77: 286-304.

[8] Findley, T. Scott and Frank Caliendo, (2008), "Short Horizons, Time Inconsistency, and Optimal Social Security," Working Paper.

[9] Findley, T. Scott and Frank N. Caliendo, (2013), "Interacting Mechanisms of Time Inconsistency," Forthcoming in Journal of Economic Psychology.

[10] Frederick, Shane, George Loewenstein, and Ted O'Donoghue, (2002), "Time Discounting and Time Preference: A Critical Review," Journal of Economic Literature 40: 351-401.

[11] Friedman, Milton, (1957), A Theory of the Consumption Function (Princeton University Press: Princeton, NJ).

[12] Gourinchas, Pierre-Olivier and Jonathan A. Parker, (2002), "Consumption over the Life Cycle," Econometrica 70: 47-89.

[13] Guo, Lei and Frank Caliendo, (2012), "Time-Inconsistent Preferences and Time-Inconsistent Policies," Working Paper.

[14] Huang, Huaxiong, Moshe A. Milevsky, and Thomas. S. Salisbury, (2012), "Optimal Retirement Consumption with a Stochastic Force of Mortality," Insurance: Mathematics and Economics 51: 282-291.

[15] İmrohoroğlu, Ayşe, Selahattin İmrohoroğlu, and Douglas H. Joines, (2003), "Time-Inconsistent Preferences and Social Security," Quarterly Journal of Economics 118: 745-784.

[16] Laibson, David, (1997), "Golden Eggs and Hyperbolic Discounting," Quarterly Journal of Economics 112: $443-477$.

[17] Leland, Jonathan W., (2002), "Similarity Judgements and Anomalies in Intertemporal Choice," Economic Inquiry 40: 574-581.

[18] Marín-Solano, Jesús and Jorge Navas, (2009), "Non-Constant Discounting in Finite Horizon: The Free Terminal Time Case," Journal of Economic Dynamics and Control 33: 666-675. 
[19] Myerson, Joel and Leonard Green, (1995), "Discounting of Delayed Rewards: Models of Individual Choice," Journal of the Experimental Analysis of Behavior 64: 263-276.

[20] Noor, Jawwad, (2009), "Hyperbolic Discounting and the Standard Model: Eliciting Discount Functions," Forthcoming in Journal of Economic Theory.

[21] Park, Hyeon S., (2009), "Bounded Rationality and Life-Cycle Consumption: A General Equilibrium Model with Short Term Planning," Working Paper.

[22] Park, Hyeon S., (2012), "Present-Biased Preferences and the Constrained Consumer," Working Paper.

[23] Rabin, Matthew, (2002), "A Perspective on Psychology and Economics," European Economic Review 46: $657-685$.

[24] Richard, Scott F., (1975), "Optimal Consumption, Portfolio and Life Insurance Rules for an UncertainLived Individual in a Continuous-Time Model," Journal of Financial Economics 2: 187-203.

[25] Rubinstein, Ariel, (1988), "Similarity and Decision-Making Under Risk (Is There a Utility Theory Resolution to the Allais Paradox?)" Journal of Economic Theory 46: 145-153.

[26] Rubinstein, Ariel, (2003), "Economics and Psychology'? The Case of Hyperbolic Discounting," International Economic Review 44: 1207-1216.

[27] Salanie, Francois and Nicolas Treich, (2006), "Over-Savings and Hyperbolic Discounting," European Economic Review 50: 1557-1570.

[28] Samuelson, Paul A., (1937), "A Note on Measurement of Utility," Review of Economic Studies 4: $155-161$.

[29] Strotz, R. H., (1956), "Myopia and Inconsistency in Dynamic Utility Maximization," Review of Economic Studies 23: 165-180.

[30] Yaari, Menahem, E. (1965), "Uncertain Lifetime, Life Insurance and the Theory of the Consumer," Review of Economic Studies 32: 137-150. 